\title{
A Novel RFID-Based Thermal Convection Type Inclinometer with Nonfloating Structure and Xenon Gas*
}

\author{
Jium-Ming Lin ${ }^{1}$ and Cheng-Hung Lin ${ }^{2}$ \\ ${ }^{1}$ Department of Communication Engineering, Chung-Hua University, Hsin-Chu, 30012 Taiwan \\ ${ }^{2} \mathrm{D}$ Ph. D. Program in Engineering Science College of Engineering, Chung-Hua University, Hsin-Chu, 30012 Taiwan \\ \{jmlin \& b09306014\}@chu.edu,tw
}

\begin{abstract}
This research proposes a novel wireless RFID-based thermal convection inclinometer. Six new ideas are presented. The first one is to make it on a flexible substrate to reduce the energy dissipation through the traditional silicon. The second one is to integrate both an inclinometer and a wireless RFID antenna on the same substrate, such that it becomes a wireless sensor and very convenient for usage. The third idea is to apply a hemi-cylindrical chamber instead of the previous rectangular one, so the gas distribution is more streamlined to increase the sensitivity. The fourth idea is to apply xenon gas instead of the traditional carbon dioxide; the latter may oxidize the heater and the thermal sensors. The fifth idea is to use a nonfloating structure instead of the floating one, thus it is more simple and low cost without making a cavity in the substrate. The sixth idea is to apply a stacking material for the temperature sensors, so that the sensitivities are better. Three kinds of stacking height were studied, such as $1,1.5$ and $2 \mathrm{~mm}$. Moreover, three types of material with different thermal conductivity are also tested, such as alumina nitride (AlN), copper and silicon. One can see the combination of AlN/2mm is better, and the sensitivity curve is approximate to a theoretical sinusoidal one.

Index Terms - Flexible substrate, Inclinometer, RFID-based, Thermal convection, Wireless sensor.
\end{abstract}

\section{Introduction}

The inclinometers can be applied in many fields, such as automobile industry for car alarms, active car suspension, and seats monitoring. In other types of vehicle, such as golf carts, forklifts, tractors or caterpillar tracks, fleet management can include monitoring of inclination to reduce the danger of rolling over or flipping due to improper handling, driving on steep slopes or exceeding load limits. In recent, it is wildly applied in the mobile phone to detect the attitude for image display. Conventional inclinometers were manufactured on silicon wafers [1-12]; some of them applying thermal convection are simpler in structure [1-7]. Six new ideas are presented in this paper. The first new idea is to make the device on a flexible substrate, thus it can save more energy than the traditional silicon. The second new idea is to integrate both a thermal convection type inclinometer and a wireless RFID antenna on the same flexible substrate as in Fig. 1, such that it is being a wireless sensor and very convenient for usage. The third idea is to use a hemi-cylindrical chamber instead of the previous rectangular one, so the gas distribution is more streamlined to increase the sensitivity. The fourth idea is to apply xenon gas instead of the traditional carbon dioxide; the latter may oxidize the heater and the thermal sensors. The fifth idea is to use a nonfloating structure instead of the floating one, thus it is more simple and low cost without making a cavity in the substrate. The sixth idea is to apply a stacking material for the temperature sensors, so that the sensitivities are better. Three kinds of stacking height were studied, such as $1,1.5$ and $2 \mathrm{~mm}$. Moreover, three types of material with different thermal conductivity are also tested, such as alumina nitride (AlN), copper and silicon. If the chamber is filled with the xenon gas, one can see the combination of AlN/2mm is better, and the sensitivity curve is approximate to a theoretical sinusoidal one. The paper organization is as follows: the first section is the introduction. The second one is fabrication steps. The third one is simulation and discussion. The last part is the conclusion.

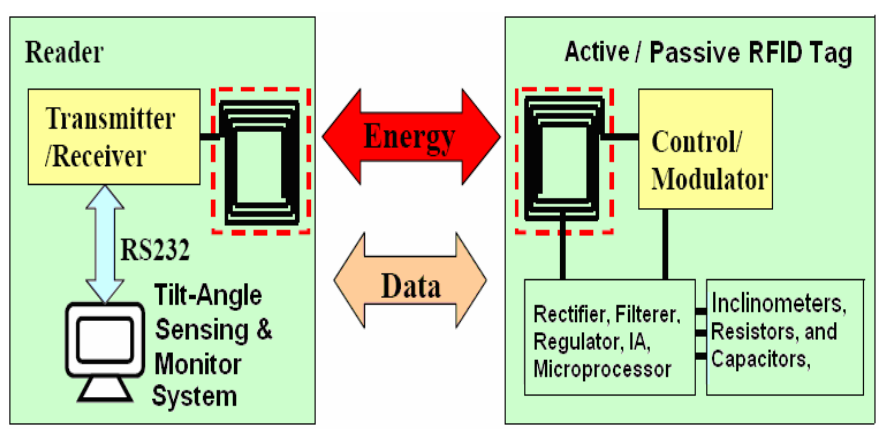

Fig. 1 Block diagram of inclinometer module.

\section{Fabrication Steps}

Step 1: Deposit $\mathrm{SiO}_{2}$ on substrate for thermal, electrical and humidity isolation. Cover Photo Resist (PR) to protect the layers of $\mathrm{SiO}_{2}$. Cover a layer of SU-8 PR at the front side. Use mask \#1 and Photolithography And Etch Processes (PAEP) to make a cavity on substrate. The result is as in Fig. 2.

Step 2: Deposit $\mathrm{SiO}_{2}$ on the substrate as the sacrificial layer; the thickness is about one half of the SU-8 layer. Cover a layer of PR on the front side. Use mask \#1 and PAEP, the $\mathrm{PR}$ on the cavity structure is remained to protect $\mathrm{SiO}_{2}$. Then remove the layer of $\mathrm{SiO}_{2}$ around the cavity.

* This work is partially supported by National Science Council Taiwan, R.O.C. with contract NSC-101-2622-E-216-001-CC3, 101-2221-E-216-006-MY2, and 101-2221-E-216-019-.to Jium-Ming Lin. 


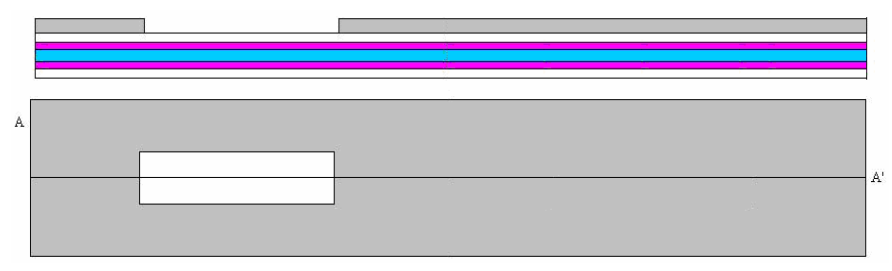

Fig. 2 Result of design Step 1.

Step 3: Remove PR on the cavity structure. Deposit a layer of alumina oxide to support the heater and temperature sensors. Cover a layer of SU-8 PR, and use mask \#2 and PAEP, only remain the PR on the grooved structure to protect the under lying layer of alumina oxide. The result is in Fig. 3.

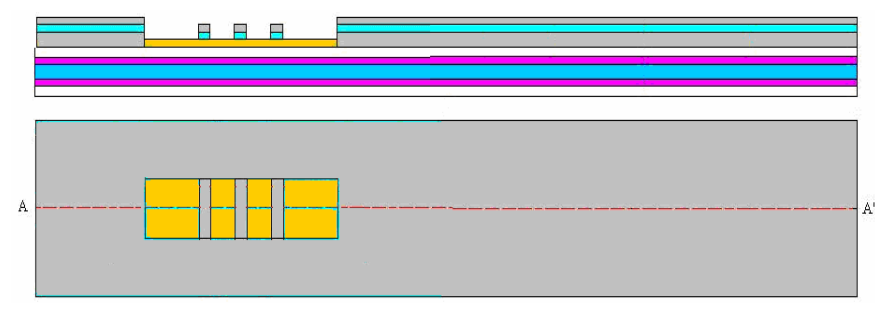

Fig. 3 Result of design Step 3.

Step 4: Deposit a layer of p-type amorphous silicon with thickness $100-250 \mu \mathrm{m}$, use an Nd-YAG laser to anneal it as a poly-silicon thermister. Then use mask \#2 and PAEP to reserve the PR on the poly-silicon thermister. Finally, use $\mathrm{KOH}$ solution to remove the layers of poly-silicon without PR protection. Remove the PR, the result is as shown in Fig. 4.

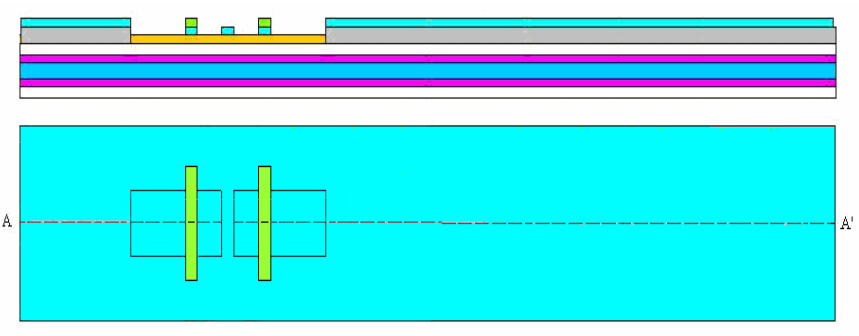

Fig. 4 Result of design Step 4.

Step 5: Deposit layers of $\mathrm{Cr}$ and $\mathrm{Ni}$ as heater, RFID antenna, and the conductors connected to the power supply. Use mask \#4 and PAEP to reserve the PR on the heater, RFID antenna, and the conductors connected to the power supply. Finally, use sulfuric acid solution to remove the layers of $\mathrm{Cr}$ and $\mathrm{Ni}$ without PR protection. After remove the PR, the result is as shown in Fig. 5.

Step 6: Use mask \#5 and PAEP to reserve the PR on the heater, and flash a layer of gold on Ni by electroless-plating. Thus the conductivity of the RFID antenna, and the conductors connected to the power supply are very good. Besides, the packaging performance of the soldering process on the pads would be increased. The result is as shown in Fig. 6.

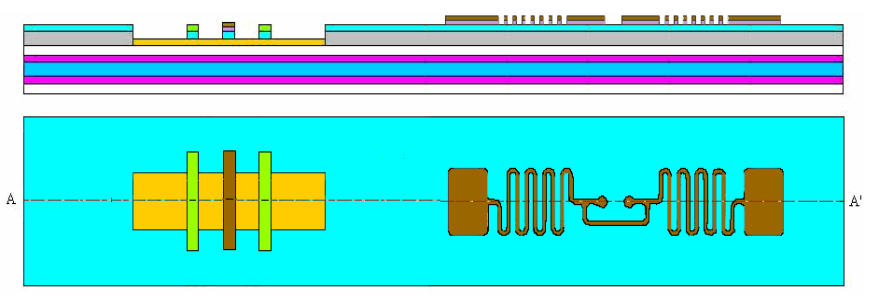

Fig. 5 Result of design Step 5.

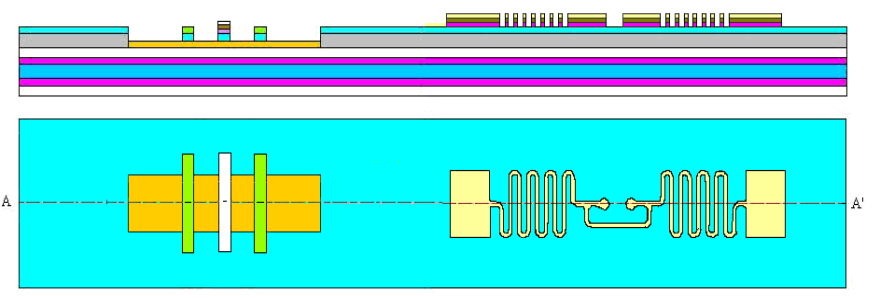

Fig. 6 Result of design Step 6.

Step 7: Cover PR on the front side. Use mask \#2 and PAEP to make the silicon dioxide layer uncovered by the PR at the cavity structure. Remove the dioxide layer, then the heater and the thermisters can be released and suspended over the cavity structure, the result is as shown in Fig.7.

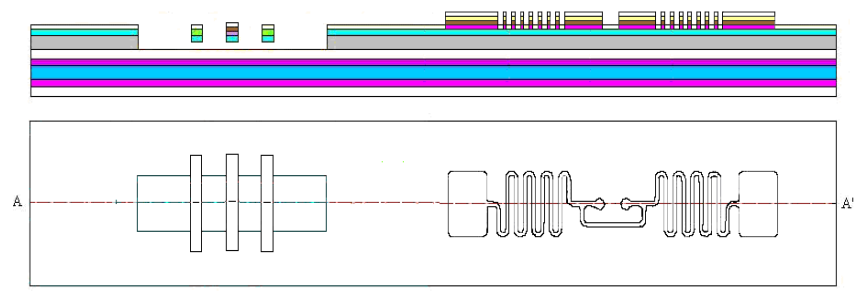

Fig. 7 Result of design Step 7.

Step 8: Remove PR, and screen print plastic material around the inclinometer as dam bar, then put a cap on the dam bar and curing, before sealing fill it with xenon gas. Finally, the chip is flip chip bonded to the RFID feed terminal, then the result is as shown in Fig. 8 with the hemi-cylindrical chamber.

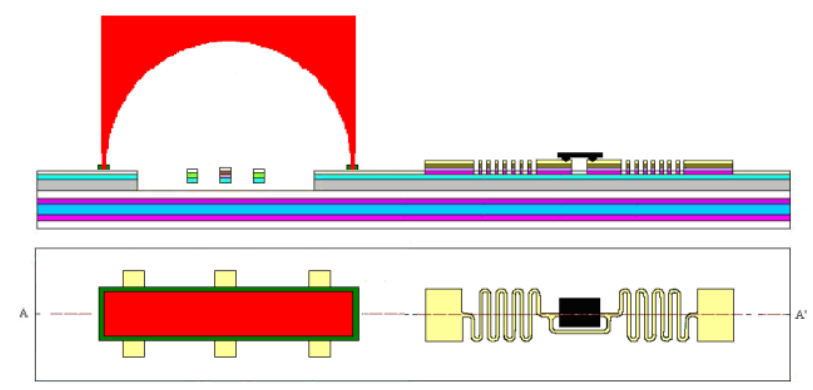

Fig. 8 Inclinometer with hemi-cylindrical chamber.

Step 9: Put a socket and/or a spring to fix a battery on the substrate, the result is as shown in Fig.9, in which a full differential bridge sensor circuit is applied. 


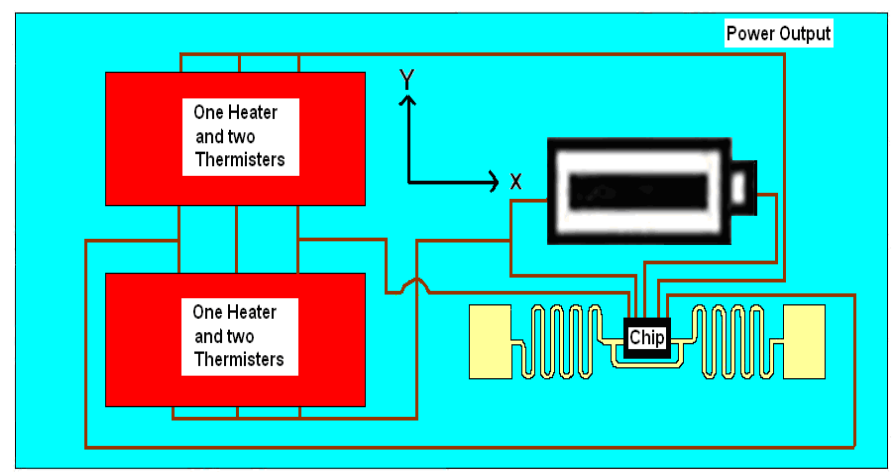

Fig. 9 Result of design Step 9.

It should be noted that if we need a nonfloating type structure without the cavity, then one can skip the related cavity making steps, such as steps 1-3 and 7 .

\section{Simulation and Discussion}

In this section we use ESI-CFD ${ }^{+}$software for simulation. Let the length and the floating height (H1) of thermisters and heater are $9 \mathrm{~mm}$ and $1 \mathrm{~mm}$, respectively. The inclinometer geometry is as shown in Fig. $10(\mathrm{~W} 1=0.5 \mathrm{~mm}, \mathrm{~W} 2=1 \mathrm{~mm}$, $\mathrm{S}=1.25 \mathrm{~mm}$ and $\mathrm{H}=8.7 \mathrm{~mm}$ ). Besides, the temperature of the package boundaries and the heater are set as 300 and $400^{\circ} \mathrm{K}$, respectively. If the chamber is filled with xenon gas, then the sensitivity curves (temperature difference at the center points of thermisters versus the tilt angle $\theta$ ) are as shown in Fig. 11. Note that the performance is not good, so the nonfloating structure as shown in Fig. 12 is applied in this paper.

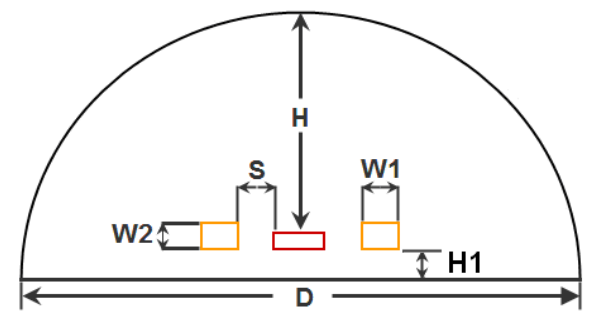

Fig. 10 Floating type inclinometer geometry.

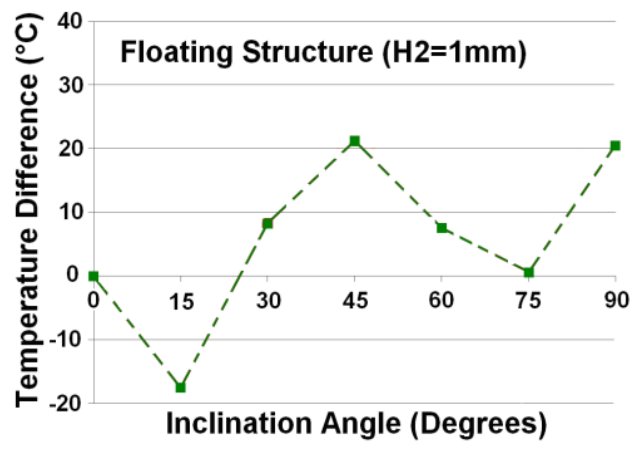

Fig. 11 Floating type inclinometer sensitivity curves.

Moreover, to increase the sensitivity, the thermisters are stacked on one material as shown in Fig. 12, in which
$\mathrm{W} 1=0.5 \mathrm{~mm}, \mathrm{~W} 3=0.5 \mathrm{~mm}, \mathrm{~S}=1.25 \mathrm{~mm}$ and $\mathrm{H}=9 \mathrm{~mm}$. The stacking material can be anyone of alumina nitride, copper and silicon. Besides, three values of stacking height $\mathrm{H} 2$, e.g. 1, 1.5, and $2 \mathrm{~mm}$, are tested for comparison. The sensitivity curves for various stacking materials and heights are as shown in Figs. 13(a), 13(b) and 13(c), respectively. Note that the case by using AlN and $\mathrm{H} 2=2 \mathrm{~mm}$ is better as shown in Fig. 13(c); the sensitivity curve is approximate to a theoretical sinusoidal one.

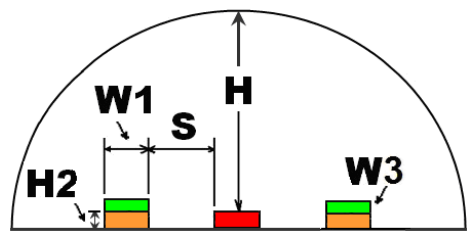

Fig. 12 Nonfloating type inclinometer geometry.

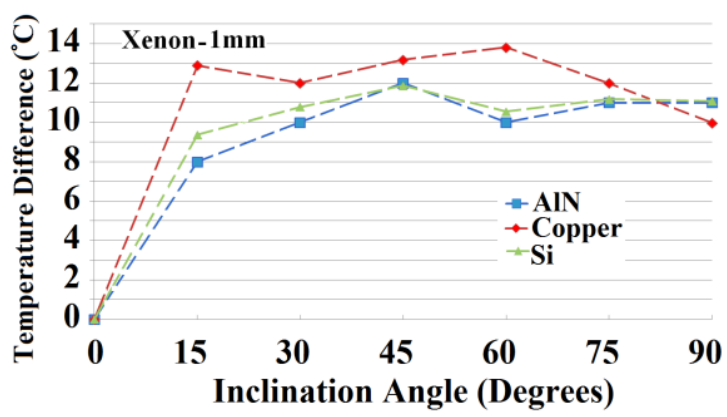

(a) $\mathrm{H} 2=1 \mathrm{~mm}$

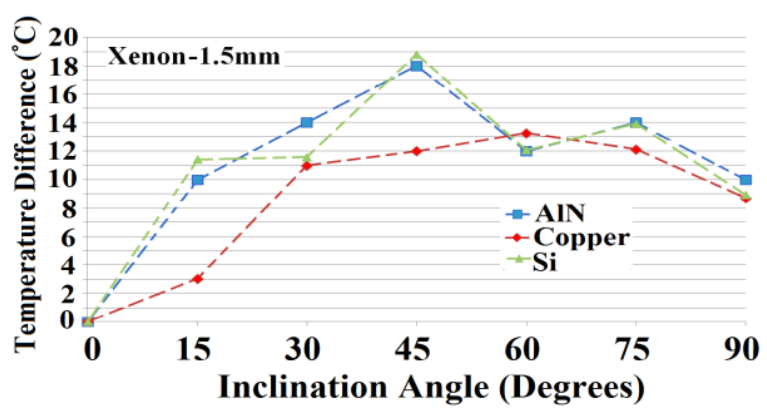

(b) $\mathrm{H} 2=1.5 \mathrm{mmm}$.

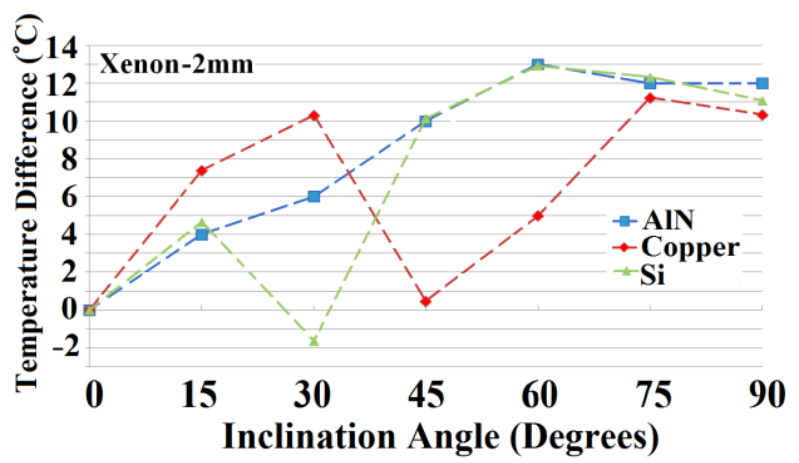

(c) $\mathrm{H} 2=2 \mathrm{~mm}$.

Fig. 13 Sensitivity curves with various stacking materials and heights. 
Besides, to show the reason by stacking a material under the thermisters to increase their temperature difference and the sensitivity, the distributions of temperatures and velocity vectors for the tilt angles $(\theta)$ as $45^{\circ}$ and $75^{\circ}$ are respectively as shown in Figs. 14(a) and 14(b) and Figs. 15(a) and 15 (b). Moreover, since the value of specific heat for AlN is the largest and the thermal conductivity is the moderate ones as shown in TABLE I that the energy and the temperature at the thermister can be easily accumulated and kept, and then the sensitivity performances would be better.

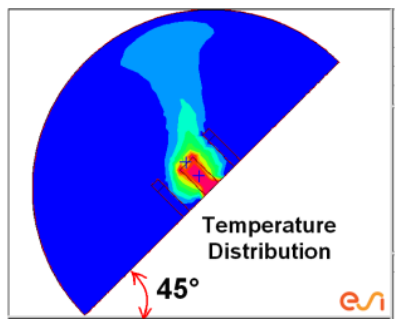

(a) Temperature distribution.

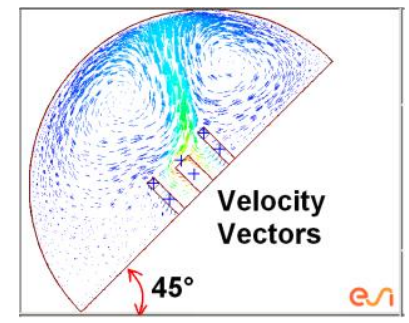

(b) Velocity distributions.
Fig. 14 Distributions of temperatures and velocity vectors $\left(\theta=45^{\circ}\right)$.

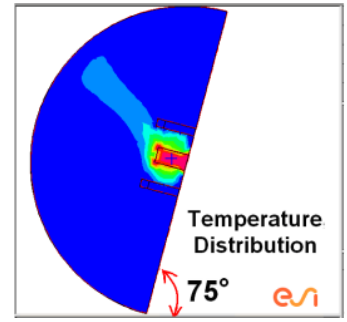

(a) Temperature distribution

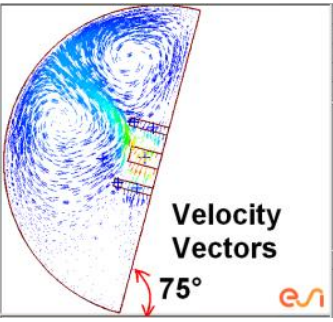

(b) Velocity distribution.
Fig. 15 Distributions of temperatures and velocity vectors $\left(\theta=75^{\circ}\right)$.

TABLE I Thermal Conductivity and Specific Heat of Materials

\begin{tabular}{|l|c|c|}
\hline \multirow{2}{*}{ Stacking Materials } & \multicolumn{2}{|c|}{ Thermal Properties } \\
\cline { 2 - 3 } & Thermal Conductivity & Specific Heat \\
\hline Aluminum nitride & 160 & 740 \\
\hline Copper & 385 & 385 \\
\hline Silicon & 124 & 702 \\
\hline
\end{tabular}

\section{IV . Conclusion}

This research proposes a novel wireless RFID-based thermal convection type inclinometer design, and relates more particularly to manufacture it on a flexible substrate. Six new ideas are presented. The first new idea is to make the device on a flexible substrate to reduce the energy dissipation through the traditional silicon. So we can present a second new idea to integrate both an inclinometer and a wireless RFID antenna on the same substrate; it is very convenient for usage. The third idea is to apply a hemi-cylindrical chamber instead of the previous rectangular one, so the gas distribution is more streamlined to increase the sensitivity. The fourth idea is to apply xenon gas instead of the traditional carbon dioxide; the latter may oxidize the heater and the thermal sensors. The fifth idea is to use a nonfloating structure instead of the floating one, so it is much simpler and less expensive without making a cavity in the substrate. The results by using floating and nonfloating structures are studied the first; note that the sensitivity performances are not good for the former. So the non-floating structure is applied in this paper. The sixth idea is to apply a stacking material for the temperature sensors, so that the sensitivities are better. Three kinds of stacking height were studied, such as $1,1.5$ and $2 \mathrm{~mm}$. Moreover, three types of material with different thermal conductivity are also tested, such as alumina nitride (AlN), copper and silicon. One can see the combination of $\mathrm{AlN} / 2 \mathrm{~mm}$ is better, and the sensitivity curve is approximate to a theoretical sinusoidal one.

\section{Acknowledgment}

This research was supported by National Science Council Taiwan, R.O.C. with contract NSC-101-2622-E-216-001-CC3, 101-2221-E-216-006-MY2, and 101-2221-E-216-019-. Besides, we are grateful to the National Center for Highperformance Computing (NCHC) for computer time and facilities of ESI-CFD ${ }^{+}$software package.

\section{References}

[1] S. Billata, H. Glosch, M Kunze, F. Hedrich, J. Frech, J. Auber, H. Sandmaier, W. Wimmer, and W. Lang, "Micromachined inclinometer with high sensitivity and very good stability," Sensors and Actuators A: Physical, vol .97-98, no.1, pp. 125-130, April 2002.

[2] S. Billat, H. Glosch, M. Kunze, F. Hedrich, J. Frech, J. Auber, H. Sandmaier, W. Wimmer, and W. Lang, "Convection based micromachined inclinometer using SOI technology," MEMS 01, pp. 159-161, January 2001.

[3] J. Courteaud, P. Combette, N. Crespy, G. Cathebras, A. Giani, "Thermal simulation and experimental results of a micromachined thermal inclinometer," Sensors and Actuators A: Physical, vol. 141, no. 2, pp. 307-313, February 2008.

[4] Y. Zhao and YY. Cai, "Temperature-gradient cancellation technique and device," US Patent US 7,862,229 B2 January 2011.

[5] A.H. Ma, A.M. Leung, "Three-axis thermal accelerometer based on buckled cantilever microstructure," IEEE Sensors, pp. 1492-1495, October 2008.

[6] A.M. Leung, J. Jones, E. Czyzewska, J. Chen, and B. Woods, "Micromachined accelerometer based on convection heat transfer," MEMS 98, pp. 627-630, January 1998.

[7] V. Milanovic, E. Bowen, Nim Tea, J. Suehle, B. Payne, M. Zaghloul, and M. Gaitan, "Convection based accelerometer and tilt sensor implemented in standard CMOS," MEMS 98, pp. 487-490, November 1998.

[8] M.A. Adams, P. Dolan, C. Marx, and W.C. Hutton, "An electronic inclinometer technique for measuring lumbar curvature," Clinical Biomechanics, vol. 1, no. 3, pp. 130-134, August 1986.

[9] O. Frazão, R. Falate, J.L. Fabris, J.L. Santos, L.A. Ferreira, and F. M. Araújo, "Optical inclinometer based on a single long-period fiber grating combined with a fused taper," Optics Letters, vol. 31, no. 20, pp. 29602962, October 2006.

[10] A.G. Butler, D.G. Green, and R.E. Nagle, "Inclinometer," US patent US 4912662 A, March 1990.

[11] B. Alain, R. Alain, V. Bernard, G. Alain, "Thermal accelerometer with reduced sensitivity to an external magnetic field," European Patent EP1550874 B1, June 2010.

[12] G. Daia, M. Li, X.P. He, L.M. Du, B.B. Shao, and W. Su, "Thermal drift analysis using a multiphysics model of bulk silicon MEMS capacitive accelerometer," Sensors and Actuators A: Physical, vol. 172, no. 2,pp. 369-378, December 2011. 